DOI: 10.1515/awutp -2015-0109

\title{
NON CONTACT VIBRATION MEASUREMENTS ON PARABOLIC SURFACE ANTENNA
}

\author{
Dorin Simoiu, Liviu Bereteu \\ Mechanical and Vibration Department, "Politehnica" University of Timişoara, \\ Mihai Viteazu, nr.1, 300222 Timişoara, Romania
}

\section{Article Info}

Received: 29.03 .2013

Accepted: 16.04.2013

Keywords: Vibration measurements, parabolic antenna, sensors.

\begin{abstract}
In this paper it is present a non contact method for $3 \mathrm{D}$ measurements on parabolic surface antenna. In order to obtain modal parameters and modal shapes of a parabolic surface antenna a Microflown USP (Ultimate Sound Probe) is used. Microflown sensors are close to antenna and can be used for the non contact measurement of the normal component of the structural velocity.
\end{abstract}

\section{Introduction}

One of the difficult problems at vibration measurement for lightweight structures, such as the reflector parabolic antenna, is that by placing a seismic accelerometer on a structure it is appear a structural change with deviations of frequency and modal shapes of that structure.

Possible solutions to remove this inconvenience are related to the use of micro seismic accelerometers very lightweight, few tens of grams, or use of optics, in particular those with lasers and possibility of the scan of the entire structures. In both cases equipment are expensive, especially those with laser scanning.

\section{The principle of Microflown Sensors}

The basic principle of Microflown sensors is the same as the hot wire anemometer. The wire heated to a temperature of between $200^{\circ} \mathrm{C}$ and $400^{\circ} \mathrm{C}$ changes its resistance, under the influence of current fluidic what wash the wire. It is resistive change depending on the speed of fluid which washed the heated wire, decreasing the temperature of wire and thus its resistance which is connected in an electrical circuit of measurement. Microflown sensor uses two wires F1 and F2 (fig. 1), (dimensions: $5 \mathrm{~mm}$ long, $1 \mathrm{~mm}$ wide and $200 \mathrm{~nm}$ thick) and was made with nanotechnology (micromachining). 
Fluid particles moving around the two wires take the heat from the two wires through convection, upstream wire F1 transfer more heat than downstream wire F2. In this way is created a temperature difference between the two wires. Because of that temperature difference appears a difference between their resistances R1 and R2.

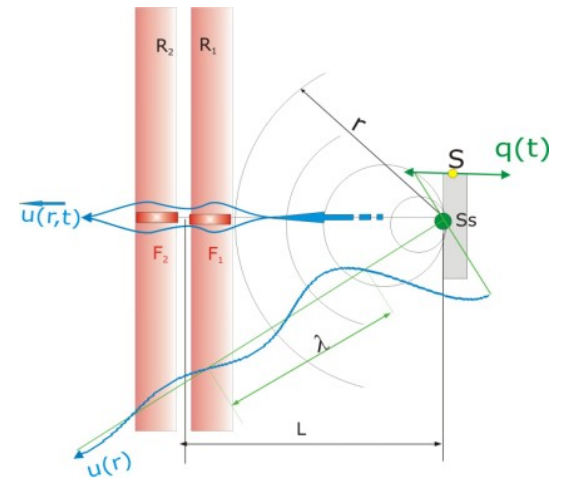

Fig. 1. Constructive principle of the Microflown sensor

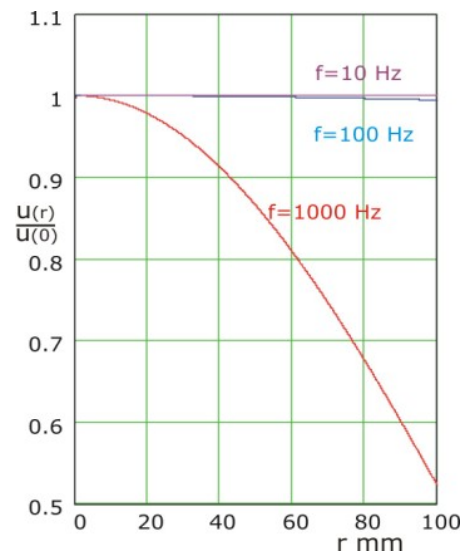

Fig. 2. Radial velocity distribution of air particles radiated by the source $\mathrm{S}$

For a spherical acoustic source Ss, the pressure wave on the sphere of radius $\mathrm{r}$ has distribution law:

$$
p(r, t)=\operatorname{Re}\left\{\frac{A e^{i(\omega t-k r)}}{r}\right\}
$$

the law of particle velocities is:

$$
u(r, t)=\frac{-1}{\rho_{0}} \int \frac{d}{d r} p(r, t) d t=\frac{A}{i \omega \rho_{0}}\left(\frac{1}{r}+i k\right) \frac{e^{i(\omega t-k r)}}{r} ; \quad i=\sqrt{-1}
$$

where $\mathrm{A}$ is a constant that depends on the intensity of the source, $\rho_{0}$ is fluid density, $\mathrm{k}$ is the wave number $\mathrm{k}=2 \pi / \lambda=2 \pi \mathrm{f} / \mathrm{v}$ ( $\mathrm{v}$ - wave propagation speed; $\lambda$ - wavelength, $\mathrm{f}$ - frequency of wave).

If the source of pressure wave is a vibrant area $\mathrm{S}$, which vibrates after a harmonic law:

$$
q(t)=q_{0} \sin (2 n f t)
$$


will radiate a pressure wave which has oscillation velocity expressed by the approximate relationship:

$$
u(r, t)=U(r) \sin (2 \pi f t)
$$

where the spatial shape of the wave is:

$$
U(r)=U(0) \frac{\sin (k r)}{r}
$$

Graphical representation of fraction $\mathrm{U}(\mathrm{r}) / \mathrm{u}(0)$ (fig. 2) shows a good propagation especially for low frequency emissions, where attenuation is practically zero for frequencies below $100 \mathrm{~Hz}$. If Microflown sensor is placed at a distance $\mathrm{L}=10 \mathrm{~mm}$ attenuation is negligible.

\section{Non-contact vibration measurement, applied to the dish antenna}

For the laboratory test we used sensor type USP (Ultimate Sound Probe) (fig. 3.), which has a compact design, with the possibility of measuring velocities of fluid particles in three directions (3D). The rectangular measuring head, where are located the three sensors has small size, $(5 \times 5 \times 5 \mathrm{~mm})$, so that allows use in narrow spaces. One of the three sensors, for example $\mathrm{S}_{1}$, consists of a closed framework fixed on one side of the rectangular head so that it forms a window $\mathrm{Fe}_{1}$ where the airflow enters, of speed $\mathrm{u}_{1}(\mathrm{t})$, a result due to movement of surface $\mathrm{P} 1$, by law $\mathrm{q}(\mathrm{t})$. The two hot wires $\mathrm{F}_{1}$ and $\mathrm{F}_{2}$ are placed inside the window $\mathrm{Fe}_{1}$ which has the role to create a nozzle tube for increase the speed $\mathrm{u}_{1}(\mathrm{t})$, having amplifier role.

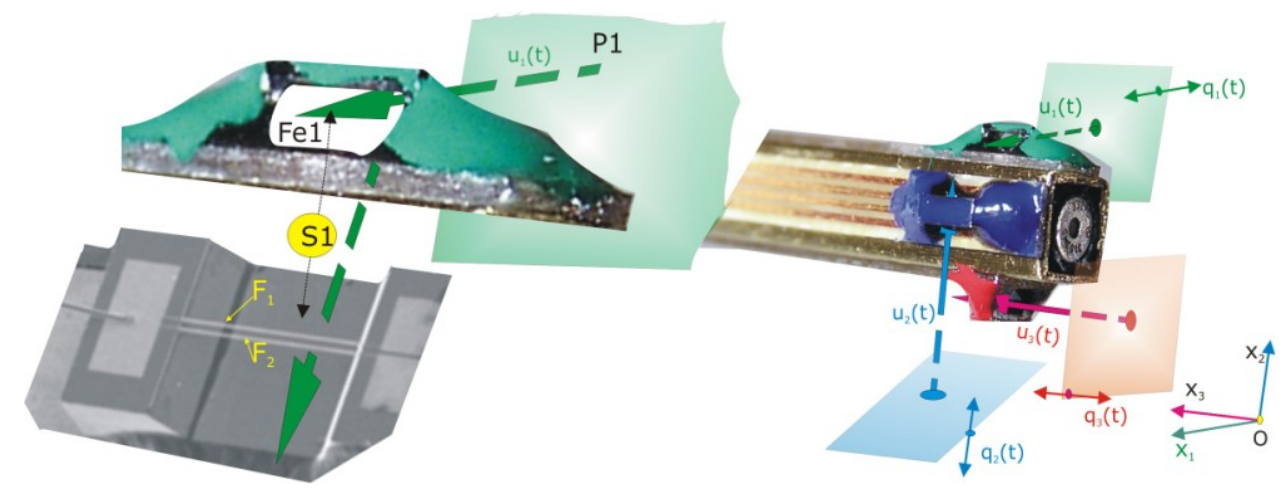

Fig. 3 Construction of USP sensor for 3D measurement

The problems raised by the correct measurement of vibration after three orthogonal directions, on a light structure like parabolic dish, are difficult, therefore was designed, made and experimented the following method.

For measurement of the particle velocity vector orthogonal components $\mathrm{v}_{\mathrm{x}}, \mathrm{v}_{\mathrm{y}}$ and $\mathrm{v}_{\mathrm{z}}$ an aluminum ring is used. It is attached by wax in points along the surface of the parabolic antenna, see fig. 4 and fig. 5 . 
At spatial movement, by vibration, of the reflector 1 , the surfaces $S_{1}, S_{2}$ and $S_{3}$ move air layers from their proximity, initiating elastic waves that propagate along normal of the surface.

The head of the USP assembly, is located inside the ring 2, with its sensors position after the normal of the surfaces $S_{1}, S_{2}$ and $S_{3}$, through adjustable support 4 and the ruler 5 attached to the support of stand 6 .

In this way, during the antenna vibrations, particles of air in the vicinity of hot wire of the three sensors, will wash these wires changing their temperatures and their resistance. The signals which results to the outputs of amplifiers are proportional to the speeds $u_{1}, u_{2}$ and $u_{3}$ of particles of the air and which are proportional to the components $\dot{q}_{1}, \dot{q}_{2}$ and $\dot{q}_{3}$ of absolute velocity of the reflector 1 in the point of attachment of ring.

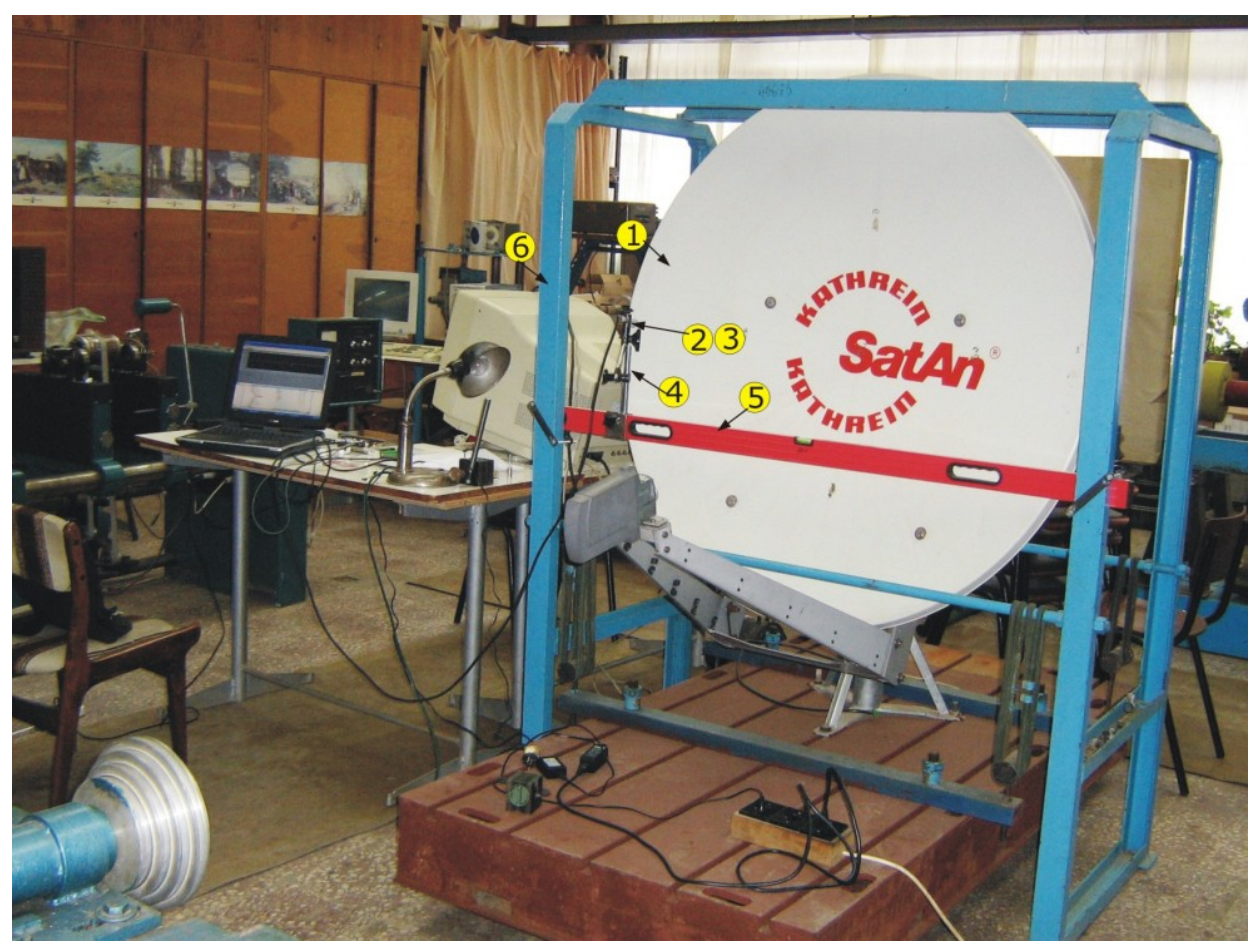

Fig. 4: Overview of the test stand 


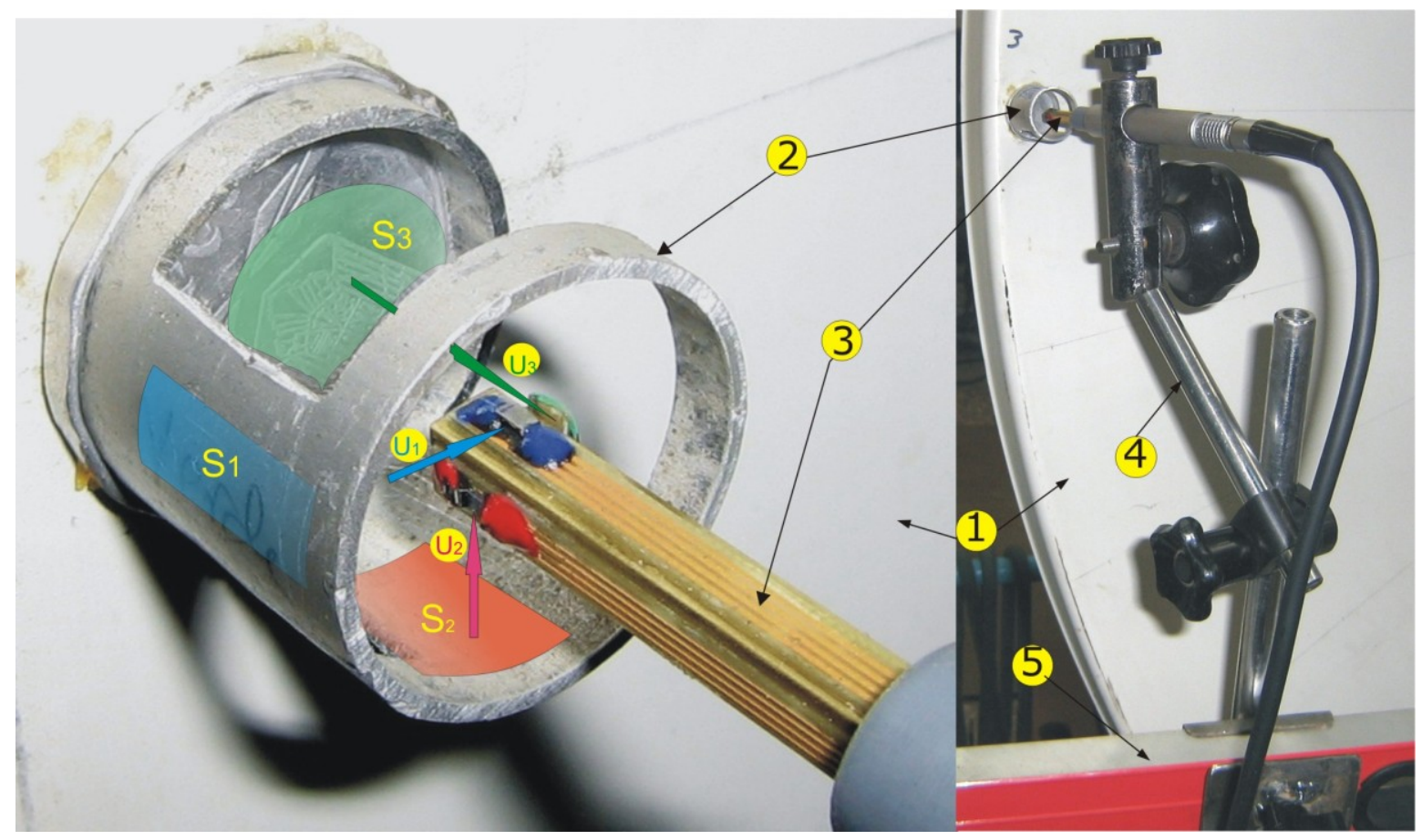

Fig. 5: A ring is used to extract the 3D velocity of a parabolic antenna

In view to demonstrate that USP sensors can be used for modal parameter and modal shapes of complex surfaces and light structures, the excitation of the antenna structure was performed by manual impulsive force, simply knocking of surface in different points of the structure. The result is shown in fig. 6 .

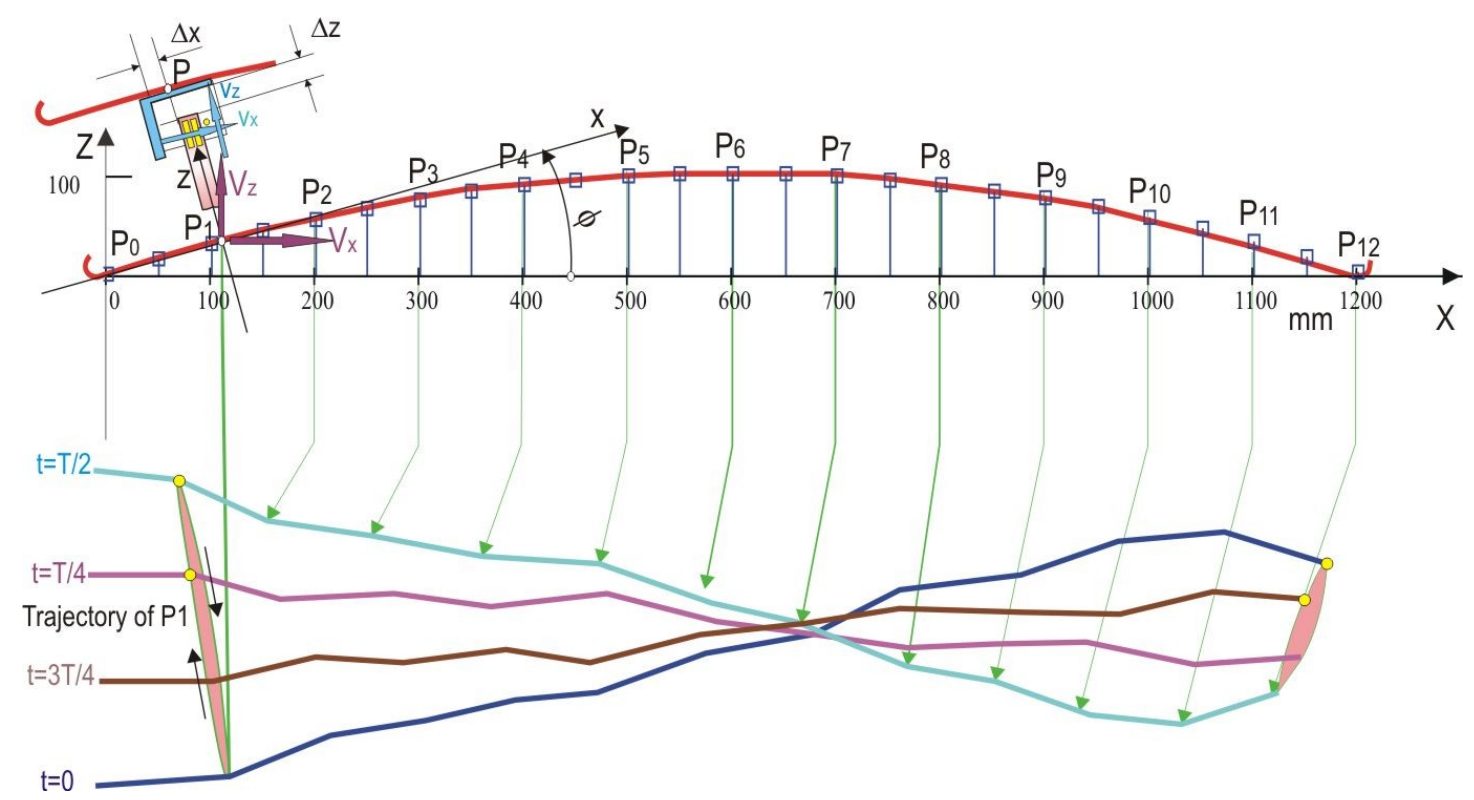

Fig. 6. Modal shape of a parabolic antenna for natural frequency $\mathrm{f}=14.2876 \mathrm{~Hz}$ 


\section{Conclusions}

- the sensitivity of the Microflown sensor is very good, the conditioner output signal has enough level for a general data acquisition system

- the solution to use the USP sensor to measure vibration in 3D by a small ring can be considered as an alternative to other solutions more expensive as using laser beam

- the solution can be improved designing a mechanical setup which provide a ease axed the USP and ring, normal to surface and to protect the sensor against mechanical damage

- more experimental testing is necessary for the suitable geometry of the ring to minimize the cross influence between sensors

\section{References}

[1] D. Simoiu, "Theoretical and Experimental Studies on Mechanical Structures Loads Induced by Wind. Application on Parabolic Dish Antenna”. PhD Thesis, University "Politehnica" of Timişoara 2008, ISBN 978-973-625-645-5. Politehnica Press.

[2] T. Cioara, First results of the USP integrated sound probe application to the 3D vibration measurements on parabolic surface antenna, University Politehnica of Timisoara, internal report, 2006

[3] T. Cioara, L. Bereteu, Determination of wind-induced dynamic loads in the structure of a parabolic antenna, Proceedings of the X-th conference on mechanical vibration, Scientific Bulletin of the „Politehnica” University of Timişoara, Tom 47(61), Supplement, (2002) 